Cahiers de recherches médiévales

Journal of medieval studies

1 | 1996

Croisades et idée de croisade à la fin du Moyen Âge

\title{
L'armement occidental pendant la première croisade
}

Olivier Bouzy

\section{(2) OpenEdition \\ 12 Journals}

Édition électronique

URL : https://journals.openedition.org/crm/2511

DOI : 10.4000/crm.2511

ISSN : 1955-2424

Éditeur

Honoré Champion

Édition imprimée

Date de publication : 15 janvier 1996

Pagination : 15-44

ISSN : 1272-9752

Référence électronique

Olivier Bouzy, "L'armement occidental pendant la première croisade », Cahiers de recherches

médiévales [En ligne], 1 | 1996, mis en ligne le 04 février 2008, consulté le 15 décembre 2022. URL:

http://journals.openedition.org/crm/2511; DOI : https://doi.org/10.4000/crm.2511

Tous droits réservés 


\section{L'armement occidental pendant la première croisade}

Lorsqu'en 1096, les croisés, Francs ou Latins, se présentèrent à Constantinople, ils entrèrent du même coup en contact avec deux civilisations qui n'étaient pas totalement inconnues en Europe, mais dont la majorité des croisés n'avait qu'une très vague idée : les Byzantins et les musulmans.

Une opinion assez généralement répandue veut que les croisés représentaient une puissance irrésistible, que leur armement défensif les transformait en des sortes d'engins blindés, insensibles aux flèches, avançant pesamment et inexorablement jusqu'à la destruction totale de l'ennemi. D'autre part, leur nombre, et leur valeur au combat les auraient rendus invincibles. En face, des Byzantins pusillanimes sont considérés comme incapables de résister aux attaques de quelques Turcs. Quant à ces derniers, tout juste bons à massacrer la croisade des paysans sans défense, ils auraient été incapables de résister à l'armée des chevaliers. Le ton est alors donné, la croisade n'était en fait qu'une promenade militaire, la victorieuse progression des croisés vers Jérusalem n'étant ralentie que par les querelles entre chefs et par leur appétit de conquête, exacerbé par la découverte d'un pays opulent. Il est à peine question d'une résistance armée.

\section{Les forces en présence}

\section{Les croisés}

Or la réalité est toute autre: d'une part, les croisés, nobles ou paysans, ne furent jamais sans peur ni sans reproche: pillages et massacres commencèrent bien avant leur arrivée en Grèce, et les Turcs ne furent pas les seuls à lutter contre. La croisade des paysans s'était déjà faite sévèrement étriller par le roi Coloman de Hongrie, qui avait exterminé les bandes dirigées par les comtes Emich von Leiningen et Hartman von Dilligen, en raison des ravages sans nombre qu'elles avaient commis sur ses terres. A son arrivée en Grèce, la croisade des paysans s'était livrée au pillage de la ville de Nish, ce qui lui avait valu de sévères représailles ordonnées par l'empereur Alexis $I^{\text {er }}$ Comnène. L'empereur avait ensuite fait embarquer cette troupe hétéroclite en direction de l'autre rive du Bosphore, à Civitot, où Etienne de Blois atteste que la flotte impériale la ravitaillait nuit et jour. Certains auraient voulu continuer sans tarder et se seraient fait massacrer, leurs ossements desséchés étant rassemblés en un grand amas. Mais le massacre ne fut cependant pas aussi total que les historiens musulmans, notamment Ibn-al-Qalanissi, le laissent entendre : quand l'armée des chevaliers atteignit Civitot, elle y trouva Pierre l'Hermite et Guillaume le Charpentier, ainsi que des pauvres innombrables.

Cahiers de Recherches Médiévales (XIIT-XV์ s.), 1, 1996 


\section{Les Byzantins}

D'autre part, en fait de Grecs apeurés, les croisés tombèrent sur une armée byzantine expérimentée, forte d'environ 70.000 hommes, capable des mancuvres les plus complexes, qui défit en un tour de main toutes les troupes franques qui adoptèrent une attitude agressive, comme celle de Raymond de Saint-Gilles, qui fut sévèrement battue à Rodosto. L'armée grecque encadra assez solidement les Francs dans leur traversée du territoire impérial, sans toutefois pouvoir empêcher Godefroy de Bouillon de piller Selymbria. Evidemment, cet encadrement fut facilité par le fait que l'armée des chevaliers arrivait divisée en quatre groupes, qui purent être maitrisés l'un après l'autre. Cette armée byzantine avait toutefois un point faible: ses effectifs étaient de renouvellement délicat. Elle était de plus en plus composée de mercenaires étrangers, dont des Petchenègues, des Turcs et des Francs, les Grecs se tenant à l'écart de la carrière militaire, de plus en plus considérée comme coûteuse et ennuyeuse'.

\section{Les musulmans}

La valeur et la manoeuvrabilité de l'armée byzantine pouvait laisser prévoir que les troupes turques, qui la surclassaient numériquement, seraient des adversaires encore plus coriaces. A priori, tous les Turcs, ou Turcomans, étaient des archers redoutables ; ils ne représentaient toutefois qu'une très faible proportion de la population de la Palestine. Mais l'aristocratie était traditionnellement guerrière, comme celle des Francs, et toute la population musulmane était censée pouvoir prendre les armes contre l'agresseur, même si sa valeur au combat était assez faible. Toutefois la première rencontre tourna indubitablement à l'avantage des croisés : les Turcs furent défaits à Dorylée le $1^{\text {er }}$ juillet 1097. Le chef turc Kilij Arslan, qui avait déjà attaqué les paysans près de Civitot, tendit une embuscade aux croisés et attaqua avec ardeur une troupe de force sensiblement égale aux siennes. Du moins pensait-il avoir affaire à tous les croisés, alors qu'il ne s'agissait que de l'avant-garde, commandée par Bohémond de Tarente, suivie de fort près par deux troupes, l'une dirigée par Godefroy de Bouillon, l'autre par Raymond de Saint-Gilles. Sur le point d'être complètement enveloppé et écrasé, Kilij Arslan se réfugia à Konya.

Mais la résistance ne cessa pas pour autant, car les croisés furent dans l'incapacité d'anéantir les fuyards réfugiés dans les montagnes environnantes. Les musulmans furent même à deux doigts d'anéantir les croisés à Antioche. Chez ceux-ci, les pertes furent terribles. Même si les chiffres sont fantaisistes, les chroniqueurs s'accordent pour dire qu'en arrivant en Anatolie les croisés étaient innombrables, mais qu'à leur arrivée à Jérusalem, on les comptait fort bien. Il semblerait que les neuf dixièmes des croisés aient péri en cours de route, soit du fait des combats, soit à cause de la famine, soit à cause des conditions d'hygiène épouvantables. Nombreu-

\footnotetext{
${ }^{1} \mathrm{M}$. Kaplan, «La place des soldats dans la société villageoise byzantine (VII $-\mathrm{X}^{e}$ siècles)», Le combattant au Moyen Age, Paris (S.H.M.E.S.), 1991, pp. 45-62.
} 
ses avaient également été les désertions, dans la piétaille comme dans l'aristocratie, principalement lors du siège d'Antioche: Etienne, comte de Blois, avec toutes ses troupes, Tatikios le représentant d'Alexis Comnène, mais aussi Guillaume le Charpentier et, pour un temps, Pierre l'Ermite lui-même.

Un élément joua en faveur des croisés : la dispersion des effectifs musulmans, du fait de leurs divisions politiques. L'Orient pouvait peut-être paraître opulent aux yeux de croisés originaires d'un Occident encore peu développé ; en fait, il avait été durement éprouvé par la conquête seldjoukide, et ne s'en relèverait jamais complètement. D'autre part, les Seldjoukides étaient des nomades, et à la suite de leur conquête ils favorisèrent les pâturages plutôt que la culture. Les villes d'Orient pouvaient encore paraître fabuleusement grandes aux yeux des Occidentaux, mais elles étaient désormais entourées de régions semi-désertiques, où les croisés allaient avoir le plus grand mal à se ravitailler. Certains mangèrent chevaux, chiens, et même les cadavres de leurs ennemis. L'Egypte fatimide avait elle-même subi une famine qui coûta la vie à une bonne moitié de sa population en 1065-1072. Quant aux divisions des musulmans, elles étaient de type religieux depuis la séparation entre chiites et sunnites, et politique depuis les débuts de la dynastie abbasside. Celleci était d'ailleurs passée sous la protection des Seldjoukides depuis le milieu du XI ${ }^{e}$ siècle. Mais les Seldjoukides eux-mêmes, après une série de conquêtes en Asie Mineure conduites par Malik-Chah jusqu'à sa mort en 1092, étaient considérablement affaiblis par l'indépendance de fait de nombreux émirs et attabegs, aggravée par les rivalités qui existaient entre eux. Ainsi la tentative de reprise de la ville d'Antioche, menée en 1098 par l'attabeg de Mossoul, Kerboga, finit-elle piteusement lorsqu'une partie de l'armée musulmane se débanda dès le début de la bataille. D'après le chroniqueur Ibn-Al-Athir, le ressentiment de nombreux émirs face à l'attitude insolente de Kerboga fut la cause de ce désastre. D'autre part, l'Egypte était aux mains de la dynastie Fatimide, chiite, hostile aux Seldjoukides et aux Abbassides de rite sunnite. Les Fatimides profitèrent même de l'avance des croisés pour reprendre d'assaut Jérusalem en août 1098, avant que les croisés ne l'assiègent à leur tour en juin 1099. Par ailleurs l'action de la secte des Ismailiens, notamment le groupe des Assassins, et l'instabilité dynastique des Fatimides et des Seldjoukides paralysèrent parfois durablement le pouvoir musulman. Mentionnons également l'existence de la secte musulmane des Druzes, qui ne joua toutefois pas un grand rôle à cette époque. Enfin, de nombreuses villes récemment conquises, comme Nicée, dont les Seldjoukides s'étaient emparé quinze ans avant la croisade, contenaient des populations chrétiennes grecques ou arméniennes, qui par fidélité dynastique aux Byzantins ou par zèle religieux favorisèrent la reconquête des chrétiens. L'émir Ifthikar-Edaulé, gouverneur égyptien de Jérusalem, ressentit de ce fait la nécessité de chasser les chrétiens de la ville avant l'arrivée des croisés.

Les croisés et la guerre

Les pertes au combat

\footnotetext{
${ }^{2}$ T. Bianquis, «Les pouvoirs de l'espace ismaīlien», Etats, sociétés et cultures du monde musulman médiéval, t. I, Paris, 1995, p. 109.
} 
Malgré, ou à cause de cette situation, la progression des croisés fut très lente : deux ans et deux mois entre l'arrivée en Asie Mineure et la prise de Jérusalem. Si cette avance fut facilitée par le désordre politique de la Palestine, elle fut ralentie par ce même désordre: les princes locaux laissaient passer les croisés parfois sans aucune résistance, mais ils étaient capables d'entretenir une guérilla dangereuse sur les arrières après le passage du gros des troupes. Ainsi le vaincu de Dorilée, Kilij Arslan, réussit-il à massacrer systématiquement tous les renforts croisés passant par l'Anatolie. Il n'y avait pas d'unité dans la défense, mais pas de capitulation généralisée non plus. Les villes ne pouvaient guère compter sur un secours de leurs voisins, mais elles étaient difficiles à atteindre par des voies mal entretenues et parfois impossible à prendre par des armées trouvant péniblement à se ravitailler. Enfin, la résistance parfois acharnée des musulmans qui comptaient dans leurs rangs des archers turcomans d'une redoutable habileté entrava considérablement la progression des Francs. Tout compte fait, la croisade fut loin d'être la promenade militaire que l'on croit. Dans Antioche, les combats furent même extrêmement durs, au point qu'il fallut six mois aux croisés pour refaire leurs forces et remonter leur cavalerie. Les croisés ne réussirent pas à s'emparer d'Arca, malgré un siège de quatre mois, échouèrent devant Emesse et devant Acre. Le résultat des combats, en termes de pertes humaines, est difficile à appréhender, car les textes sont contradictoires: Etienne de Blois prétendait ainsi que les croisés n'eurent aucun mort à déplorer lors de la bataille de Dorylée; mais il est vrai qu'il écrivait alors à sa femme et qu'il est rare de voir mentionner dans ce genre de littérature, qui se veut rassurante, les pertes réellement infligées par l'ennemi. De son côté, Ibn-al-Athir mentionne la désertion en masse de l'armée de Kerboga devant Antioche : on est donc relativement surpris de lire ensuite que quelques milliers de musulmans restèrent sur place envers et contre tout, et se firent massacrer. Un article de Jean Flori ${ }^{3}$ nous donne des indication sur ce que les pertes liées directement aux combats ont pu être, et elles furent apparemment loin d'être négligeables. Mais il ne semble guère possible de les définir précisément, moins parce les chiffres sont fantaisistes lorsqu'il s'agit des grandes batailles que parce qu'ils ne sont sans doute pas connus des chroniqueurs en ce qui concerne les combats de rencontre, accrochages divers et razzias. Or ces accrochages semblent former une part importante de la vie quotidienne du croisé qui dépend entièrement du pillage pour sa remonte et son ravitaillement. Le prince Ousâma ibn Mounqidh (1095-1187) fait ainsi état d'engagements sans aucune importance stratégique, mais quasi quotidiens. Ousâma eut l'occasion de rencontrer les Francs à l'occasion de plusieurs ambassades et apprit à les connaître. Ses écrits nous permettent d'appréhender de manière plus précises les conditions dans lesquelles s'affrontaient les croisés et leurs adversaires, puisqu'il fut à même de comparer les coutumes des deux camps 4 .

${ }^{3}$ J. Flori, «la valeur des nombres chez les chroniqueurs du Moyen Age», Le Moyen Age, $\mathrm{n}^{\circ} 3-$ 4, 1993, t. XCLX, pp. 416-421.

4 Ousâma, un prince syrien face aux croisés, éd. A. Miquel, Paris, 1986, passim. 


\section{Les tactiques des croisés vues par le prince Ousâma}

De façon assez curieuse, ce qui frappa surtout Ousâma chez les Francs, ce ne fut pas leur manière de combattre, ni leur armement ; ce fut avant tout leurs pratiques médicales, religieuses et juridiques. Ses conclusions sont d'ailleurs peu flatteuses: les Francs sont stupides, perfides et d'une cruauté inhumaine. Leurs mours sociales, qu'il juge très relâchées parce qu'ils laissent une certaine autonomie à leurs femmes et parce qu'ils ne sont pas très pudiques, le plongent dans des abîmes de perplexité : il ne parvient pas à comprendre comment les chrétiens peuvent être à la fois si extraordinairement braves et apparemment si peu soucieux de leur honneur. Sur leur tactique, leur hiérarchie, leur armement, il donne en passant quelques indications: les chevaliers représentent l'élite de l'armée, ils assurent la cohésion sociale des croisés, imposant de manière collégiale leurs solutions à tous les problèmes et conflits. Il y a aussi des sergents à cheval, et des sergents à pied, dont la valeur militaire est très dépréciée.

Dès le début de la conquête, les croisés employèrent des Turcopoles, qu'Ousâma définit comme des archers de mère grecque et de père musulman. A l'occasion de petits engagements, il observa des troupes fortes de 120 à 500 hommes, composées pour une moitié ou jusqu'à deux cinquièmes de fantassins ou de Turcopoles. Il assista aussi à des engagements plus importants, près de villes, où la population assiégée fournissait un complément d'au moins plusieurs milliers de fantassins, sans grande valeur. Il indique d'ailleurs à cette occasion que les Francs pratiquaient couramment la fuite simulée, de façon à attirer un ou des imprudents à l'écart du gros de la troupe, ou pour dissocier l'infanterie de la cavalerie adverse. Il observe à l'inverse que les Francs attaquent toujours groupés, sauf dans le cas de quelque tête chaude. Il donne comme évident que le sort de ces imprudents est d'être rapidement jeté à terre et capturé, quelque soit leur camp, citant comme un exemple tout à fait remarquable qu'un croisé isolé ait pu se tirer d'affaire malgré la grêle de coups qui avait abattu son cheval et dont il était lui-même accablé.

Enfin, du point de vue de l'armement, il décrit les cottes de mailles franques comme étant plus longues que celles des musulmans. Il signale toutefois qu'un coup de lance peut percer de part en part cette cotte et le cavalier qui ne peut se protéger de son bouclier, et indique au passage que le meilleur moyen de porter un coup mortel est de tenir la lance coincée entre le bras et le corps. Il considère comme normal que tous les cavaliers soient équipés d'une cotte de maille, mais signale des cas fréquents où les combattants négligent de la porter, soit pour être plus à l'aise, soit parce qu'ils n'ont pas pris le temps de la revêtir. Il signale de terribles blessures causées par les épées franques, des coups portés à la figure ayant tranché chairs et os au point qu'une partie de la face pend en avant, avec le nez et la joue presque entièrement détachés. Il rapporte enfin que Tancrède de Hauteville, prince d'Antioche, considérait comme normal que ses chevaliers surclassassent en toutes circonstances les fantassins adverses, même au prix de la perte de leur monture, et qu'il fit chasser ignominieusement deux chevaliers qui avaient été mis en fuite par un seul cavalier musulman. En fin de compte, on n'a pas l'impression que les façons de combattre des Francs et des Arabes aient été vraiment différentes. Notons toutefois qu'Ousàma 
était arabe, et qu'il nous décrit la façon de combattre des Francs en la comparant à sa propre pratique du combat au corps à corps; alors que s'il avait été turc, il aurait opéré par harcèlement mené par des archers à cheval, et ses commentaires auraient alors été différents.

\section{La guerre vue par les Francs}

\section{Les tactiques}

Si l'on se rapporte aux documents occidentaux écrits ou figurés, on trouve une confirmation partielle de ces dires, avec des nuances parfois non négligeables. Ainsi, cinq-cents combattants semble être un chiffre minimal pour une troupe envoyée en reconnaissance ou en razzia, on trouve par exemple ce chiffre de cinq-cents pour les gardes du roi dans Le roman d'Alexandre ${ }^{5}$. En-dessous, il ne peut être question que de troupes réunies en catastrophe pour s'opposer à une razzia ennemie.

Les chevaliers combattaient en groupe compact, ou mesnie, rassemblée autour du seigneur qui la commandait. A la guerre comme au tournoi, l'étude de M. Duby sur Guillaume le Maréchal confirme que l'homme seul est en effet une proie facile pour un groupe ${ }^{6}$, quoique les chansons de geste soient pleines d'exploits individuels, et de formidables coups d'épées qu'on ne devait pas avoir l'occasion d'admirer au sein d'une bataille rangée. Mais il ne faut pas négliger l'impression subjective du combattant, auditeur de la chanson de geste : si le fait littéraire souligne l'exploit individuel, c'est évidemment parce que, même au sein d'un groupe structuré, pour autant d'ailleurs que ce groupe garde sa cohésion tout au long du combat, des hommes se battant à l'arme blanche devaient parfois se sentir bien seuls face à leur ennemi. Et même si le coup d'épée le plus formidable pouvait passer inaperçu au sein de la bataille, ses résultats étaient encore visibles après.

La fuite simulée mentionnée par Ousâma est attestée déjà lors de la bataille de Hastings. Elle semble d'ailleurs le résultat d'une manœuvre fortuite, les Normands ayant observé, à la suite d'une débandade de leur armée, que les Saxons lancés à leur poursuite étaient plus facilement massacrés que lorsqu'ils étaient en ligne. Nous reviendrons d'ailleurs, à propos du bouclier et de la charge à la lance, sur les innovations imputables aux Normands en matière militaire. Trente ans plus tard, il n'est pas impossible que cette innovation se soit répandue dans les armées chrétiennes. Mais d'un autre côté, il ne faut pas oublier que lorsqu'Ousâma parle des tactiques adverses, il décrit principalement celles des Normands de Tancrède de Hauteville. La tactique de la fuite simulée n'était peut-être pas répandue chez tous les croisés.

\section{Utilisation de la lance}

L'iconographie montre que les cottes de mailles franques, si elles étaient plus longues que les cottes musulmanes, étaient toutefois encore assez courtes : elles descendaient jusqu'aux genoux, et ne couvraient parfois le bras que jusqu'au coude. El-

${ }^{5}$ Le roman d'Alexandre, L. Harf-Lancner éd., Paris, coll. Lettres gothiques, 1994, v. 4909. p. 604.

${ }^{6}$ G. Duby, Guillaume le Maréchal, Paris, 1984, passim. 
les tendent toutefois à s'allonger, mais ne couvriront entièrement le combattant qu'à la fin du XII ${ }^{e}$ siècle. Tous les combattants n'en portaient pas, mais il s'agissait alors plutôt de fantassins. La question reste d'ailleurs posée de savoir si les combattants allaient au combat sans armure parce qu'ils n'avaient pas les moyens de s'en procurer une ou parce qu'ils considéraient que l'embarras qu'elle causait n'était pas justifié par la protection relative qu'elle apportait. En effet, l'arme par excellence du combattant du $\mathrm{XI}^{e}$ siècle est la lance, et les textes confirment qu'un coup de lance pouvait transpercer un homme malgré sa cotte de mailles et que ce coup était mortel dans presque tous les cas. On peut alors admettre que l'agilité ait pu être préférée à une protection relative mais embarrassante. Non que ces cuirasses aient été extrêmement pesantes, mais elles n'étaient pas très élastiques, par nature, et gênaient les mouvements. On comprend également d'où venait la stupéfaction d'Ousâma lorsqu'il apprit qu'un croisé qu'il avait transpercé «par le travers des hanches» avait survécu à sa terrible blessure? ${ }^{7}$. Il s'agissait véritablement d'un miraculé, compte tenu de la gravité du coup reçu et de l'incapacité des médecins francs.

D'un autre côté, l'iconographie nous apprend que l'escrime à la lance couchée en arrêt sous l'aisselle n'est apparue qu'au milieu du $\mathrm{XI}^{e}$ siècle - c'est la tapisserie de Bayeux qui représente pour la première fois cette façon de tenir la lance - et qu'elle n'était pas encore généralisée lors des croisades. Ici encore, il s'agit d'une pratique introduite par les Normands. La pratique la plus répandue était de brandir la lance au dessus de la tête, la pointe baissée vers l'avant. Cette position ne permettait certes pas de transpercer à coup sûr une cotte de mailles, mais elle permettait de frapper latéralement, par la manche de la cuirasse, l'adversaire qui levait le bras. Le roi Robert $\Gamma^{\mathrm{er}}$ aurait été tué de cette façon en 923 :

Ille vero letali ictu accepto, ab eo in dextram obliquatur et sic per loricam manicam lancea eum in latere gravissimo ictu sauciat necnon et per epar atque pulmonem et sinitri lateris ypocundriam ferrum usque in clipeum transigit et, circumseptus $a b$ aliis, septem lanceis confossus praecipitatur diriguitque, multoque Fulbertus mox exhaustus sanguine et intercertans, mortuus cecidit ${ }^{8}$.

Cela implique que les cavaliers qui brandissaient leur lance au dessus de leur tête chargeaient en désordre, même s'ils attaquaient en masse, et qu'ils combattaient en fin de compte de manière relativement individuelle. Les coups se portaient le plus souvent à la tête, le fer de lance pouvant rester fiché profondément dans l'œil ou dans la bouche, malgré les tentatives d'extraction. Il fallait attendre que les os soient suffisamment mortifiés pour permettre l'arrachement du fer de lance, ou bien, dans le cas d'un fragment cassé dans la blessure, attendre que celui-ci ressorte naturellement après un long cheminement à l'intérieur du crâne : cela pouvait prendre quel-

\footnotetext{
${ }^{7}$ Miquel, op. cit., p.29.

${ }^{8}$ Richer, Histoire de France, éd. R. Latouche t. I, Paris, 1967, pp. 87-93 : Fulbert, frappé d'un coup mortel, se porte sur le côté droit de Robert et à travers la manche de sa cuirasse le blesse grièvement au côté avec sa lance. Le fer traverse le foie, le poumon, l'abdomen gauche et pénètre jusqu'au bouclier. Cerné de toutes parts, percé de sept lances, le roi est jeté à bas et tué raide. De son côté, Fulbert, épuisé par une grande perte de sang, ne tarde pas à tomber mort en combattant.
} 
ques années. Ces blessures aux conséquences pénibles ne sont évidemment pas mentionnées dans les chansons de geste, mais on en trouve dans les «Miracles». Un livre de Renate Hitze nous fait connaître le vocabulaire, volontairement varié et recherché, utilisé pour mieux rendre compte des effets d'un coup d'épieu ou de lance'. A lire les chansons de geste, il ne semble pas que le bouclier soit d'une quelconque efficacité pour arrêter un coup de lance, tout au plus pouvait-il ralentir le coup ou le dévier. En effet, ayant passé le bouclier, la lance pouvait ne frapper qu'une partie de la cuirasse, sans blesser le combattant. Cependant, dans la plupart des cas, le choc était encaissé pleinement par l'homme. Ayant percé la cotte de mailles, la lance continuait son chemin dans l'homme, perçait le côté, le foie, le pis, le flanc, le poumon, le coeur, l'échine, la coraille, l'entrague. Ces renseignements anatomiques sont d'ailleurs fantaisistes, ou témoigneraient d'un trajet parfois très sinueux de la lance dans l'organisme. Elle transperçait complètement l'homme dans la moitié des cas. Le combattant qui ne réussissait pas à dévier la lance de son adversaire était tué sur le coup dans plus de $80 \%$ des cas, et les blessés pouvaient encore mourir les jours suivants, même si la blessure paraissait bénigne, comme dans le cas d'un comte de Flandres cité par Suger:

Comes Flandrensis Balduinus... subito sed raro ictu in facie lancea percussus, dedignatus tantila sibi providere plaga, mori non dedignatus ${ }^{10}$.

Si la plus grande efficacité meurtrière de la lance peut nous paraître inattendue, puisque son usage disparait complètement par la suite, elle est reconnue comme telle par ceux qui ont pu encore en observer les effets. Ainsi d'après le général de Brack (1789-1850) : «La lance est l'arme blanche dont l'effet moral est le plus puissant, et dont les coups sont les plus meurtriers»"

\section{Utilisation de l'épée}

Les épées du $\mathrm{XI}^{\mathrm{e}}$ siècle sont flexibles et la pointe en est arrondie, la flexibilité de l'arme rendant impossible les coups d'estoc. Le combat à l'épée était donc uniquement composé de coups de taille, rendus encore plus meurtriers par un affûtage remarquable, qui est encore sensible aujourd'hui sur les épées les mieux conservées. La mise en garde des combattants à pied était destinée à permettre à ceux-ci de frapper l'adversaire le plus vite possible. Protégés par leur bouclier tenu à bout de bras devant eux, les combattants francs tenaient leur épée à bout de bras derrière eux, la main à hauteur de ceinture, la pointe de l'épée juste au dessus de leur tête (fig. 1). La

\footnotetext{
${ }^{9}$ R. Hitze, Studien zu Sprache und Still der Kampfschilderung in der chansons de geste, Genève, 1965, p. 49 : les verbes utilisés pour en décrire les effets dévastateurs sont rompre, démailler, trancher, despaner, fausser, desmentir, désafrer, desclore, percer, empirier, desconfire, trouer, dessertir, desserrer, fendre, délicer, desconcendre, défaire, essartir, desclaveler, desrouter et descoudre.

${ }^{10}$ Suger, Vie de Louis VI le Gros, éd. H. Waquet, Paris 1964, p. 194, chap. XXVI, (11381144) : Le comte de Flandres Baudouin... reçut sur la figure un brusque mais assez léger coup de lance; il dédaigna de prendre aucune précaution pour une plaie si insignifiante, mais la mort, elle, ne le dédaigna pas.

${ }^{11}$ A. F. de Brack, Avant-postes de cavalerie légère, Paris, 1831. Rééd. 1942, p. 57.
} 
position ne devait pas être confortable, mais elle permettait de frapper sans retard, horizontalement, vers le ventre, la poitrine ou la tête de l'adversaire (fig. 2).

Il existe encore deux autres mises en garde, moins fréquentes. Dans l'une, l'épée était brandie au-dessus de la tête, le poing devant la figure, la pointe de l'épée tournée vers l'arrière (fig. 3). Cette position devait permettre d'assener des coups verticaux, de préférence lorsque la victime était au contact, voire même à portée de la main, dans le cas d'exécutions par exemple (fig. 4). Dans la dernière position de mise en garde, la moins fréquemment représentée, la main était au niveau de la ceinture, la pointe de l'épée dirigée vers l'avant (fig. 5). Cette position était peut-être prise lorsque le combat s'éternisait ou lorsque le danger n'était pas immédiat. Elle permettait en tout cas de prendre rapidement la mise en garde préalable aux coups verticaux.

On a peu de mentions, dans les rapports de fouilles, de squelettes présentant des blessures. C'est qu'aucun charnier consécutif à une bataille n'a été retrouvé pour la période qui nous intéresse. Les crânes retrouvés avec des traces de blessures sont donc le résultat soit de petits combats locaux, soit d'échauffourées individuelles. Ces blessures ne sont pas systématiquement mortelles, et peuvent cicatriser lorsqu'elles sont de longueur inférieure à $10 \mathrm{~cm}$. Une étude porte sur 73 crânes français et étrangers montrant des traces de blessures : sur 28 traumas crâniens, $32 \%$ des blessures ont été suivies de guérison, une autre étude montre que les coups portés à la tempe représentaient $45 \%$ des blessures, les coups portés sur le front $24 \%{ }^{12}$. Un article consacré à six squelettes d'époque saxonne découverts à Eccles (Kent), permet d'affiner notre idée des pratiques de combat ${ }^{13}$. Quatre des crânes portaient des blessures causées par des coups horizontaux, la partie touchée se trouvant à l'arrière du crâne, près du conduit auditif, comme si le combattant avait instinctivement tourné la tête du côté opposé au coup. Le cervelet étant alors atteint, la victime s'effondrait immédiatement. Les deux autres montraient des coups verticaux portés sur le front. Les entailles dans les os crâniens étaient longues de 12 à 16 centimètres et avaient toutes été mortelles. On trouve ce type de blessure à la tête représenté de façon réaliste dans un manuscrit du livre des Machabées (fig.6). Dans les romans médiévaux mentionnant des blessures, les trois quarts des coups étaient effectivement portés à la tête, puis venaient l'épaule et la hanche, dans le cas où le combattant ratait son but ${ }^{14}$.

On combattait à l'épée aussi bien à pied qu'à cheval, dans ce dernier cas toutefois seulement après avoir perdu sa lance. Les combattants à cheval avaient dans l'ensemble la même mise en garde qu'à pied, main en arrière et pointe vers l'avant, ou main au-dessus de la tête, pointe de l'épée vers l'arrière. L'ennemi n'étant plus en face du combattant, mais sur son côté gauche ou droit, le frapper horizontalement par dessus la tête du cheval prenait du temps et risquait d'effrayer l'animal. On peut

\footnotetext{
${ }^{12}$ R. Perrot, «Les blessures et leur traitement au Moyen Age», Histoire et Archéologie, $\mathrm{n}^{\circ}$ 97, 1985, pp. 42-47.

${ }^{13}$ S.J. Wenham, «Anatomical Interpretation of Anglo-Saxon Weapon Injuries», Weapons and Warfare in Anglo-Saxon England, Oxford, 1989, pp. 122-139.

${ }^{14} \mathrm{P}$. A. Sigal, «Les coups et blessures reçus par le combattant à cheval en Occident aux XII et XIII siècles», Le combattant au Moyen Age, S.H.M.E.S., 1991, pp. 171-183. Egalement Hitze, op. cit., p. 54-56.
} 
donc conjecturer qu'on devait frapper horizontalement l'ennemi à sa droite, et verticalement celui à sa gauche.

Après ce bref aperçu des technique les plus usuelles, nous allons voir de quel équipement les croisés pouvaient se prévaloir face à leurs adversaires.

\section{Etat de l'armement des croisés au XI ${ }^{e}$ siècle}

\section{Armes offensives}

- Epées

La grande épée à deux tranchants est une arme chère, et d'ailleurs relativement inefficace vu son prix. Plus qu'une arme indispensable, c'est un signe distinctif du noble et riche chevalier ; tous les combattants à cheval n'en sont d'ailleurs pas équipés.

Au début du $\mathrm{XI}^{e}$ siècle, ces armes sont fabriquées dans des ateliers importants et en portent la marque. C'est le nombre des trouvailles archéologiques et leur diffusion géographique qui permet de juger de la productivité d'un atelier, et de sa réputation. Ainsi, malgré le manque désespérant d'indices, une probabilité existe pour que l'atelier INGELRII se soit trouvé à Cologne ${ }^{15}$. Les épées de cet atelier furent ensuite vendues dans toute l'Europe. Qu'on en juge : on en a trouvé à Dun-sur-Auron (près de Bourges), à Rothenburg (canton de Luzern, Suisse), Saint-Jean de Saône (près de Chalon), à Londres, à Wandsworth (près de Londres), à Tingvoll (près de Flemma, Norvège). D'autres, d'origine inconnue, sont conservées aux musées de Glasgow et Zurich. Une carte de David Illis en mentionne d'autres, mal localisées, en Allemagne, Autriche, Finlande, Russie et Suède ${ }^{16}$. Un livre d'Ada BruhnHoffmeyer et un article de Jean Pierre Arignon en mentionnent aussi, également en Suisse et en Russie.

D'autres ateliers ont également laissé leur marque :

- +ULFBERH+T

- GICELIN

- LEOFRIC

- SUNDTHVES

- CONSTAININIUS ${ }^{17}$

\section{- CEROLD \\ - ULEN \\ - LEUTFRIT \\ - LUN ${ }^{18}$.}

${ }^{15}$ Je m'appuierais pour cela sur un indice foumi par la verrière de sainte Cécile dans l'église Saint-Kunibert de Cologne, qui daterait du début du XIII ${ }^{e}$ siècle. Ce vitrail présente en effet une caractéristique remarquable : c'est la seule représentation d'épée qui porte lisiblement un nom d'atelier, en l'occurence INGELRII. Le vitrail est daté, pour autant qu'il l'ait été correctement, d'une époque où l'atelier n'est justement plus en activité. Je pense toutefois, vu la forme de l'épée, que le vitrail est plus ancien que sa date estimée, et remonte à la fin du XII ${ }^{c}$ siècle. Il est possible que les donataires, qui sont représentés au registre inférieur, aient été les propriétaires de l'atelier, alors que celuj-ci approchait de sa fin.

${ }^{16} \mathrm{D}$. Illis, The Sword in anglo-saxon England, Oxford, 1962, pl. V.

${ }^{17}$ A. Bruhn-Hoffmeyer, Middelalderens Tveäggede Swaert, Copenhague, 1954, passim.

${ }^{18}$ J.-P. Arignon, «le combattant russe, IXe-XIII siècles, d'après les données archéologiques», Le combattant au Moyen Age, S.H.M.E.S., 1991, p. 128. 
Il faut effectivement voir en ces inscriptions un nom d'atelier plus qu'un nom de personne, INGELRII se rencontrant sur les épées du $\mathrm{X}^{\mathrm{e}}$ au XI $\mathrm{XI}^{\mathrm{e}}$ siècle, avec des variantes INGELRED / FIT, INGERII T, INGERII FECIT du XI ${ }^{e}$ au XII ${ }^{e}$ siècle.

Les lames sont constituées d'un métal contenant ferrite, perlite et martensite. $\mathrm{Ce}$ mélange est de la sorbite, ce qui signifierait un recuit de l'arme après la trempe ${ }^{19}$. En fait, de minces lignes de soudure attestent que les tranchants sont toujours soudés à une âme, constituée d'un feuilletage de fers et d'aciers de duretés différentes. Les tranchants sont parfois composés du même mélange de métaux, parfois simplement renforcés d'une baguette de perlite soudée en biais sur les bords. D'après les recherches de Marco Panseri ${ }^{20}$, les forgerons soudaient des petits morceaux d'acier sur une plaque de fer doux, puis repliaient l'ensemble plusieurs fois jusqu'à obtenir une structure feuilletée qu'ils façonnaient en une barre, ébauche de la nouvelle épée. Les tranchants de l'ébauche, dont la structure moléculaire avait été modifiée par le pliage, étaient plus résistants que s'ils avaient été composés d'un empilage de tôles soudées. Deux fines plaques d'acier étaient enfin soudées sur chaque tranchant et donnaient son fil à l'épée, tout à la fois souple et résistante aux chocs. On obtient ainsi des lames qui pèsent de 500 à 900 grammes pour des longueurs de 90 à 100 $\mathrm{cm}$. Les armes complètes, garde et pommeau montés, pesaient de 1,5 à $1,8 \mathrm{~kg}$. On désigne actuellement cette technique sous le terme de damas feuilleté, quoique la surface du métal ne ressemble plus au véritable damas. La lame porte une gorge sur toute sa longueur. Cette gorge n'est pas faite pour faciliter l'écoulement du sang en cas de pénétration de la lame dans le corps, puisque l'arme ne peut pas être utilisée d'estoc. Elle existe sur les épées depuis le IVe siècle et la meilleure interprétation semble être que la dépression centrale est en fait causée par le rajout des tranchants, ce qui cause des bourrelets de part et d'autre du noyau.

Plus que la forme et la texture de la lame, c'est l'aspect de la poignée - pommeau et garde - qui permet de dater une épée. Le pommeau sert à équilibrer l'arme et permet ainsi les moulinets les plus serrés. il pèse souvent autant que la lame. C'est la partie la plus visible d'une arme qui la plupart du temps, ne l'oublions pas, est portée à la ceinture, dans un fourreau. C'est donc la partie qui sera le plus sensible aux phénomènes de mode, celle qui reflétera également le rang de son propriétaire. Il est la plupart du temps en fer, quelques rares pommeaux sont exceptionnellement dorés - dans le cas d'armes appartenant à des rois - gravés, ou décorés de motifs floraux en filigrane (fig. 7). On observe que les épées trouvées en France ont généralement au $\mathrm{XI}^{\mathrm{e}}$ siècle un pommeau lenticulaire, alors que les épées germaniques sont ornées d'un pommeau en forme de noix de cajou.

Ces armes mesurent de $95 \mathrm{~cm}$ en France à $115 \mathrm{~cm}$ en Allemagne, taille qui n'est pas déterminée en fonction d'un compromis entre les limites techniques de la résistance de l'acier et la nécessité d'une efficacité minimale, sinon elles auraient toutes la

\footnotetext{
${ }^{19}$ R. F. Tylecote et B. Gilmour, The metallography of early ferrous Tools and edged Weapons, Oxford, 1986, pp. 220-221.

${ }^{20}$ C. Panseri, «Ricerce metallographica sopra una spada del XII secolo", Documenti e contributi per la storia della metallurgia, $\mathrm{n}^{\circ} 1,1954, \mathrm{pp} .1-31$.
} 


\section{Olivier BOUZY}

même taille. Il semble que la longueur de la lame soit fonction de la taille moyenne des hommes de l'époque. L'iconographie nous montre les fourreaux attachés à la ceinture, à hauteur de hanche, la bouterolle ne touchant pas le sol, ce qui nous donne une indication de la taille des hommes de guerre, qui aurait augmenté au cours du $\mathrm{X}^{e}$ siècle, les épées passant d'une taille moyenne de $90 \mathrm{~cm}$ autour de l'an mil à $101 \mathrm{~cm}$ en 1100. La grande disparité dans la longueur des épées laisse deviner que les combattants médiévaux devaient chercher à acquérir des épées correspondant à leur morphologie. On peut également déduire des sobriquets de deux ducs de Normandie que les épées n'étaient pas faites sur mesure : Robert Courte-Heuse, qui s'illustra au cours de la croisade, et son fils Guillaume Longue-Epée. Il semble que ces ducs aient eu les jambes courtes, et que le surnom du premier vienne de la petite taille de ses chausses, tandis que celui du second viendrait de ce que son épée devait, en trấnant par terre, paraitre proportionnellement plus grande que celle des autres hommes. Mais il s'agit là d'un cas particulier: l'arme que portent ces ducs est vraisemblablement celle de Rollon, on la voit d'ailleurs entre les mains de Guillaume le Conquérant sur la tapisserie de Bayeux. C'est une arme spécifique par la forme de son pommeau - semi-circulaire, alors que les pommeaux du milieu du $\mathrm{XI}^{\mathrm{e}}$ siècle sont circulaires - c'est aussi une arme-sceptre, symbole de la continuité dynastique chez les ducs de Normandie, de même que les rois de France arborent au moment de leur sacre l'épée dite de Charlemagne ${ }^{21}$.

Ces épées portent également des inscriptions ou des invocations religieuses souvent abrégées, en plus des noms d'atelier. On trouve des motifs géométriques simples: ronds, croix et barres, des lettres faites d'un fil de fer aplati et soudé à la lame, qui ressort en clair sur le fond d'acier. On peut trouver des séries de lettres formant de véritables invocations, abrégées selon un système qui n'est pas particulier aux épées, mais qui suit les règles normales de la paléographie, exception faite du tilde qui n'est pas représenté, ou exceptionnellement. Les abréviations les plus fréquentes sont :
A : altissimus
$\mathrm{H}: \mathrm{me}$, homo
$\mathrm{B}$ : benedictus, beatus
HO : homo
C: consolator, creator, caelum
$I$ : in, Jésus
O: omnipotens
$\mathrm{D}:$ dominus, deus
IE : Jésus
DOS : dominus
$\mathrm{K}$ : Christus?
$\mathrm{R}$ : rex
S : salvator
DII : domini
$\mathrm{M}:$ me, meus
S, SCS, SES : sanctus
$T:$ fecit
$\mathrm{N}$ : in, nomine
$\mathrm{U}$ : universus
E : eternus
NE, NIOME, NOMNE : nomine
XP : Christus ${ }^{22}$
FIT : fecit

${ }^{21}$ Sur les armes symboles d'un pouvoir politique, cf. O. Bouzy, "Les armes symboles d'un pouvoir politique: l'épée du sacre, la Sainte Lance, l'Oriflamme, aux VIII'-XII ${ }^{e}$ siècles», Francia, 22/1, 1996, sous presse.

${ }_{22}$ Bruhn-Hoffmeyer, op. cit, passim, Arignon, op. cit., p. 129. 
On remarque aussi une tendance à utiliser le I au lieu du $\mathrm{E}$, le $\mathrm{H}$ comme un $\mathrm{M}$ ou un $\mathbf{N}$, une orthographe parfois fantaisiste. En fait, cela est peut-être dû simplement à une mauvaise lecture de ces inscriptions, souvent très corrodées. L'invocation in nomine domini, très répandue à partir du $\mathrm{XI}^{e}$ siècle, est parfois réduite à sa plus simple expression: «NED ${ }^{23} »$, mais prend parfois des formes plus complexes: «+INNIOMMHDII $+»^{24}, «+$ INIOMINICI $+{ }^{25}$, «+NNOMIIEDN $+»^{26}$. On peut proposer quelques interprétations des inscriptions; ainsi pour une épée conservée à Ber$\operatorname{lin}^{27}$, où l'on peut lire «NIDICOIACNUSDISMD», on peut comprendre in NomIne DominI COnsolatoris, lesus AGNUS DeI Salva Me Domine. D'autres interprétations sont sans doute possibles. Des invocations presque aussi longues sont écrites en toutes lettres: «BENEDICTUS DOS DES MEUS QUI DOCET MANUS ¿s $^{\text {s }}$ (benedictus dominus deus meus qui docet manus). D'autres sont plus laconiques: «IERS $»^{29}$ (IEsus Rex Sanctus). Toutes les épées ne portent pas d'inscription, mais les $\mathrm{XI}^{\mathrm{e}}-\mathrm{XII}^{\mathrm{e}}$ siècles figurent en tête des périodes où les épées sont le plus fréquemment décorées ${ }^{30}$.

\section{- Epieux}

Cette arme peu connue est probablement la plus répandue, aussi bien aux mains des paysans que des chevaliers... du moins dans les textes, car on en trouve peu qui soient conservées. C'était une sorte de grand coutelas à un seul tranchant, de 60 à $8 \mathrm{C}$ $\mathrm{cm}$ de long, emmanché sur une hampe qui est généralement représentée comme étant de faible longueur (fig. 8), ce qui permettait de l'utiliser comme une épée, de taille, mais aussi d'estoc, l'extrémité en étant pointue. Les chansons de geste attestent également de son utilisation comme d'une lance, ce qui suppose qu'on y adaptait le cas échéant une hampe plus longue. Cela faisait de l'épieu une arme intermédiaire entre la lance et l'épée, ce qui introduisit d'ailleurs une certaine confusion dans le vocabulaire médiéval, latin et français. Il aurait certainement supplanté complètement la lance si, à partir du $\mathrm{XI}^{\mathrm{e}}$ siècle justement, la charge à la lance couchée ne s'était peu à peu imposée. Pour cet usage, l'épieu était trop long et trop flexible, et allait complètement disparaître de l'armement des cavaliers au XII ${ }^{e}$ siècle. En revanche, il se maintint dans l'équipement des piétons.

C'était une arme d'origine germanique fort ancienne: d'après le dictionnaire F.E.W. ${ }^{31}$, le mot épieu viendrait du francique *speot, non attesté. Le dictionnaire Bloch et von Wartburg ${ }^{32}$ lui donne également pour origine le francique *speot. Il en

${ }^{23}$ Epée trouvée à Saint Omer, Berlin, Zeughaus, non numérotée, Bruhn-Hoffmeyer, op. cit., p. $11, n^{\circ} 43$.

${ }^{24}$ Epée trouvée en Italie, Copenhague, coll. Christensen C 1, Ibid. p. 8, $\mathrm{n}^{\circ}$ 7, pl. IXb.

${ }^{25}$ Zürich, Landesmuseum, 16347, Ibid., p. 10, n ${ }^{\circ} 31$, pl. IXd.

${ }^{26}$ Musée national du Danemark D 10255, Ibid., p. 12, $n^{\circ} 1$, pl. Xa.

${ }^{27}$ Berlin, Zeughaus, inv. 7370, Ibid., p. $21, \mathrm{n}^{\circ} 87$.

${ }^{28}$ Epée de Saint Maurice, attribuée à Henri III le Noir (emp. 1039-mort 1052), trésor impérial de Vienne (Autriche), Ibid., p. 12, $\mathrm{n}^{\circ} 67$.

${ }^{29}$ Zürich, Landesmuseum, AG 2465, Ibid., p. 10, n³6, pl. IXe.

${ }^{30}$ Datation des inscriptions dans l'article de Arignon, op. cit. pp. 128-133.

${ }^{31}$ Französisches etymologisches Wörterbuch, Berlin-Schöneberg, 1955.

${ }^{32}$ Dictionnaire étymologique de la langue française, P. U. F., 1975. 


\section{Olivier BOUZY}

fait également découler l'allemand moderne Spiess, l'ancien prussien espeut et l'ancien français espiet, altéré en espiel puis espieu d'après le mot pieu. D'après le dictionnaire Kluge ${ }^{33}$, le mot Spiess, allemand pour épieu, viendrait de l'ancien hautallemand spioz, issu du germanique *speuta, d'origine inconnue, qui donnerait également le vieux français espiet et la forme *speot, cette fois considérée comme anglo-saxonne. Il est à mon avis plus probable que le germanique *speuta fut recomposé d'après le grec $\sigma \pi \alpha \theta \eta$ (spathè), qui signifie littéralement «battoir à

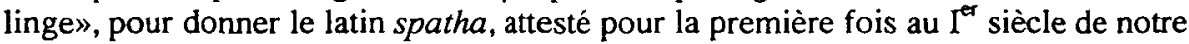
ère par Tacite (Annales, 12, 35). C'était alors une arme aux mains des troupes auxiliaires. On en trouve ensuite mention dans Végèce: Gladios majores, quos spathas vocant; et alios minores, quos semispathas ${ }^{34}$, mais elle n'apparaît pas en France avant le $\mathrm{VI}^{e}$ siècle. C'est alors une arme à un seul tranchant, qui évolue lentement vers un double tranchant, le dos étant aiguisé sur une longueur de plus en plus grande, et qui ne sera plus, dès lors, distinguée du glaive. Spatha était synonyme de gladius depuis 430, mais aussi de framea, qui est une sorte de lance : Hastas vel ipsorum vocabulo frameas gerunt angusto et brevi ferro ${ }^{35}$, et de romphea et machera. Spatha, considéré comme un mot populaire, a été traduit de préférence par ensis. On trouve en effet ce mot utilisé pour désigner une arme de jet dans Richer : Propinquantesque patrio more in primo tumultu enses jaciunt... A regius equitatus, ensium nube dilapsa ${ }^{36}$, mais aussi pour désigner clairement une épée dans l'Histoire anonyme de la première croisade : et invaserunt saracenos masculos et feminas decollantes eos nudi ensibus ${ }^{37}$. Spatha est moins utilisé en latin médiéval que gladius, et il est même remarquable, et au fond parfaitement justifié, de voir Raban Maur prétendre que spatha est un mot vulgaire :

Framea vero quam vulgus spatham vocat: ipsa est romphea... Semispathium gladius a media spatae longitudine appelatus ${ }^{38}$.

Raban Maur copiait d'ailleurs fidèlement la glose d'Isidore de Séville: Gladius ex utraque parte acutus, quam vulgus spathan vocat $t^{39}$, qui copiait lui-même celle de saint Augustin :

\footnotetext{
${ }^{33}$ Etymologisches Wörterbuch der deutschen Sprache, Berlin, 1967.

${ }^{34}$ Végèce (cf. dictionnaire Du Cange), Traité sur l'art militaire, livre 12, chapitre 15 (fin IV début $\mathrm{V}^{e}$ siècle après $\mathrm{J}$. C.).

${ }^{35}$ Tacite, Germania, 6 ( $c f$. dictionnaire Gaffiot) : Les lances, ou dans leur langage framées, portent un fer étroit et bref.

${ }^{36}$ Richer, Histoire de France, èd. R. Latouche, t. I, Paris, 1967, pp. 182-183, chap. 35 (995998 ) : Pendant la progression et au moment où commence l'engagement, ils jettent leurs [épieux] suivant la coutume nationale... Mais la cavalerie royale, le nuage des [épieux] dissipé...

${ }^{37}$ Histoire anonyme de la première croisade, éd. L. Brehier, Paris, 1924, pp. 206-207, chap. 38 (1099) : ils attaquèrent les Sarrasins, hommes et femmes, les décapitant, l'épée nue.

${ }^{38}$ Raban Maur, De Universo sive de rerum naturis, J. P. Migne, Patrologiae cursus completus, Patres latini, t. 111, col. 537a, livre XX, VI, (842-846).

${ }^{39}$ Isidore de Séville, Liber Etymologiorum, XVIII, 6 (c. 636).
} 
Framea appelatur quam vulgo spatham dicunt. Sunt enim gladii ex una parte acuti, ipsae sunt macherae ipsae autem framea, ipsae et romphea, ipsae etiam spathae appelantur ${ }^{40}$.

On peut voir d'ailleurs que c'est entre l'époque de saint Augustin et de Raban Maur, soit entre 430 et 840 , que la spatha avait acquis un double tranchant, mais plus probablement vers cette dernière date, puisque Abbon de Saint-Germain sentait encore en 897 la nécessité de préciser qu'un ensis avait deux tranchants: Hic ensis bisacutus adest meus ${ }^{41}$. Dès 881 la cantilène de sainte Eulalie atteste la forme spede : "Ad une spede li roveret tolir lo chief»" Et la Vie de saint Alexis les formes «espede», «espethe»:

strophe XV : «pois li cumandet les renges de s espethe».

strophe LXXXIII : «Espede ceindra cume tui altre per» ${ }^{43}$.

On trouve espee dans la Chanson de Roland, la Prise d'Orange, la Chanson de Guillaume, et on trouvera espaza dans la Chanson de la croisade albigeoise pour désigner une arme de jet ${ }^{44}$ :

E gietan dartz e lansas et las peiras punhaus

E cairel et sagetas e apchas e destraus

E lansas e espazas e bastos e tinaus

Spatha est donc à la fois à l'origine des mots «épée» et «épieu», ensis n'ayant pas été conservé par le vocabulaire courant.

Cette surprenante incertitude du vocabulaire est évidemment due à une certaine imprécision, de même que nous nommons fusil (du latin vulgaire foculus, pierre à feu) aussi bien les anciens fusils à platine que les modernes fusils d'assaut, et de surcroît les instruments à aiguiser les couteaux. Bien qu'on puisse aussi l'employer, on l'a vu, dès le IX siècle au plus tard pour désigner également les épées, diverses citations permettent toutefois de supposer que le mot ensis désignait de façon préférentielle les armes à un seul tranchant, et donc les sabres, aussi bien celui de Charlemagne, conservé au trésor impérial de Vienne: Aliquoties et gemmato ense utebatur, quod tamen nonnisi in praecipuis festivitatibus vel si quando exterarum gentium legati venissent ${ }^{45}$, que ceux des combattants musulmans: [Ejus] ensem vero emit quidam LX bisantis ${ }^{46}$.

\footnotetext{
${ }^{40}$ Saint Augustin, Psaumes, 149, 12 (c. 430).

${ }^{41}$ Abbon de Saint-Germain, Bella Parisiacae Urbis, éd. H. Waquet, Paris, 1964, pp 94-95, chant II, vers 385 (885-897) : Il est auprès de moi comme une épée à deux tranchants.

${ }^{42}$ La cantilène de sainte Eulalie, éd. M. P. Dion, Valenciennes, 1990, v. 22 (881).

${ }^{43}$ La vie de saint Alexis, éd. J. M. Meunier, Paris, 1933 (c. 1040).

${ }^{44}$ La chanson de la croisade albigeoise (le continuateur anonyme), éd. H. Gougaud, Paris, 1989, pp. 254-255, chap. XVII, laisse 156, vers 27 et sq. (1218-1219).

${ }^{45}$ Eginhard, Vita Caroli Magni, 23, MGH, SSRG, Hannover, 1922 : «Il prenait parfois un sabre couvert de gemmes, mais seulement lors des festivités les plus importantes, ou lorsque des ambassadeurs étrangers se présentaient».

${ }^{46}$ Histoire anonyme de la première croisade, éd. Bréhier, pp. 216-217, chap. 39 (1099): Quelqu'un acheta soixante besants le sabre [de l'émir de Babylone].
} 
Il paraît en tout cas évident que l'épieu du $\mathrm{XI}^{e}$ siècle n'a guère de rapport avec l'arme de chasse que nous désignons communément sous ce nom. Les descriptions des chansons de geste nous permettent de nous orienter vers une arme tranchante, pointue, niellée, avec une douille carrée et emmanchée $e^{47}$. De nos jours, on la trouve désignée par le nom de fauchard, mais il s'agit de la même arme, car on voit lancer le fauchard dans les chansons de geste du XII siècle comme on avait lancé l'épieu: «[à] Olivier ont lancié maint faussart esmoulu»" ${ }^{48}$.

Les plus anciens exemplaires sont mérovingiens (fig. 9). La douille est carrée, de façon certaine à partir du XIII siècle (fig. 10), et probablement auparavant, mais le petit nombre d'objets conservés ne permet pas d'établir une typologie bien fiable.

\section{- Lances}

Le fer de la lance est d'une facture souvent remarquable lors de son apogée au $\mathrm{XI}^{e}$ siècle. Il est alors généralement constitué de six parties forgées, soudées et décorées avec beaucoup de soin, dont une en damas. Le damassage forme d'abord le noyau de la flamme, servant d'articulation entre la flamme et la douille, ce qui offre une plus grande flexibilité à l'ensemble, grâce à sa structure spiralée. Il semble ensuite n'être plus utilisé que comme décor sur la flamme. La douille, quant à elle, est constituée d'une tôle d'acier enroulée en forme de cône autour de la base de la flamme.

Un élément remarquable des pointes de cette période est la présence quasi systématique d'ailettes. On pourrait penser qu'elles ont la même fonction que sur un épieu de chasse : éviter que le fer ne traverse le corps de l'adversaire. Ce n'est en fait pas le cas; ces ailettes sont faites de petites lamelles de métal, avec deux ou trois tenons, soudées dans une encoche incisée le long de la douille. Comme il est hors de question que ces faibles lamelles puissent résister à un choc un tant soit peu violent, leur fonction n'est donc que décorative. Plus exactement, il s'agit des témoins fossilisés de deux crochets de renfort placés de part et d'autre de la douille, mais qui n'avaient plus d'utilité pratique dès le début du VII' ${ }^{e}$ siècle. On peut expliquer leur permanence par le conservatisme des artisans, qui perpétuaient de façon traditionelle les formes préexistantes, même lorsqu'elles étaient devenues inutiles. On remarque en effet que les pointes de lance évoluent moins vite que les épées. Si on tient compte de ce qu'on pourrait appeler la durée de vie d'un de ces types d'armes, on note qu'il faut attendre près de 150 ans avant de constater une modification significative de la longueur de la lame, de la forme de la douille, de sa décoration ou de la taille des ailettes, alors que les épées évoluent deux fois plus vite.

Comme dans le cas des épées, le grand nombre d'exemplaires d'une même forme permet de deviner l'existence d'ateliers centraux. On peut même identifier sur la lame des marques d'artisans qui ressemblent aux marques des tailleurs de pierre. Elles se trouvent surtout sur certaines pointes de lance aux formes pratiquement standardisées, sous la forme de gravures qui sont moins des décors que des signatures. Il existe au moins trois groupes d'ateliers marqués par des différences régionales, comme pour les épées, formant non pas une, mais plusieurs chronologies. On

${ }^{47}$ Hitze, op. cit., p. 207-209.

${ }^{48}$ Fierebras, éd. F. Guessard, Paris, 1860, p. 51, vers 1680 (milieu du XII ${ }^{\mathrm{e}}$ siècle). 
trouve ainsi au $\mathrm{XI}^{\mathrm{e}}$ siècle de nombreuses pointes dont la partie de la douille située sous les ailettes est décorée d'incisions rappelant les fils de fer qui maintenaient les crochets de renfort au VII siècle. Sur les deux autres «faces», des incisions rappellent l'effet que produisaient les angles des douilles octogonales du VIII siècle. Ces incisions décorent aussi les ailettes. Ce type de lances se retrouve dans la vallée de la Saône et dans le Jura. S'il est peut-être aventureux de faire des lances à douille incisée un type spécifique bourguignon, puisque le seul indice de localisation d'un atelier dans cette région est la présence d'une manufacture d'armes à Autun au... IV siècle de notre ère, on peut néanmoins insister sur la remarquable diffusion de ce type d'arme, puisqu'on trouve des pointes ainsi décorées à Londres, au Danemark, et jusqu'en Pologne. Par ailleurs, des différences existent quant à la forme de la lame: en définissant schématiquement un triangle entre l'embouchure de la Seine, le massif central et les sources du Danube, on dira que les lances triangulaires sont plus fréquentes dans l'angle nord, les fers lenticulaires «en navette» plus nombreux dans l'angle est, les lames en « feuille de saule» plus abondantes à l'ouest. Bien sûr, ces trois pointes du triangle doivent être considérées comme le centre de zones qui s'interpénètrent et se chevauchent largement, tandis que marchandages ou guerres exportent en petite quantité et de façon aléatoire les armes loin de leur centre de fabrication.

Le risque de casser la hampe ou la douille de l'arme, qui en sont les parties fragiles, est bien réel en cas de torsion au moment du choc. En effet, parmi les fers repêchés dans le lit des rivières, on retrouve parfois un reste de hampe emmanché dans la douille, à moins que le fer ne soit cassé quelques centimètres en dessous de la flamme. Une étude réalisée sur la description des combats à la lance montre qu'une proportion importante de lances cassait en effet au premier choc ${ }^{49}$. L'analyse des restes de bois conservés dans la douille permet de dire que, dans la presque totalité des cas, la hampe est en bois de frêne. Elle s'affine en forme de cône, pour se réduire au contact du fer à un diamètre allant de deux à trois centimètres. L'usage du frêne est bien attesté par les textes : «Je te ferai cest fregne parmi le corps passer» ${ }^{50}$, mais on trouve également cité le pommier : «Ou cors li met la hante de pommier» ${ }^{51}$.

\section{Armes défensives}

\section{- Cuirasses}

Il est difficile de dire quelque chose d'assuré à propos des défenses de corps des combattants du XI siècle, étant donné qu'aucune d'entre elles n'a été conservée jusqu'à nos jours, sauf peut-être celle de Rodrigo Diaz de Bivar, le célèbre Cid Campéador, qui mourut précisément en 1099. Conservée à Burgos, cette cuirasse, pour autant qu'elle soit authentique, est composée d'écailles métalliques fixées sur une veste de cuir. C'est cela qu'on doit appeler une cotte de mailles, la maille étant une pièce de monnaie. Ce que nous appelons, nous, cotte de mailles, était en fait désigné par l'expression «cotte treslie». L'iconographie ne nous est pas, dans ce cas particu-

\footnotetext{
${ }^{49}$ Sigal, op. cit., pp. 171-183.

${ }^{50}$ Fierebras, éd. Guessard, p. 16, vers 504 (milieu XII' siècle).

${ }^{51}$ Otinel, éd. Guessard, Paris, 1859, p. 59, v. 1690 (fin XII' siècle).
} 


\section{Olivier BOUZY}

lier, d'un grand secours. Quelques dessins représentant les cottes sous l'aspect de rangées d'ondulations horizontales toutes rangées dans le même sens, doivent en effet figurer des cottes de mailles. D'autres, représentant des ondulations verticales, rangées alternativement dans un sens puis dans un autre, doivent figurer des cottes treslies. Mais on ne peut pas déduire grand chose de dessins représentant des cottes hachurées de petits carreaux en oblique, parfois de couleurs variées. Quoiqu'il en soit, ces cottes, broignes ou loricae, sont assez courtes, s'arrêtant au milieu de l'avant-bras et à mi-cuisse. Elles sont complétées par une coiffe, ou haubert, sorte de cagoule qui, étymologiquement, protège le cou, et qui finira également par désigner la cuirasse entière. Ce capuchon n'est pas fixé sur la cotte, ce qui n'est pas très visible sur les enluminures, mais peut se déduire des textes. Ces cottes ne sont sans doute pas très lourdes, mais elles sont assez rigides, surtout dans le cas des cottes de mailles, et sont extrêmement pénibles à supporter, même pour des hommes très entraînés. Guillaume de Poitiers nous décrit ainsi Guillaume le Conquérant portant sur son épaule, au soir d'une reconnaissance à cheval, la lorica de Guillaume FitzOsbern, pourtant "célèbre par sa force ${ }^{52}$. L'Histoire anonyme de la première croisade nous montre également des chevaliers incapables d'emporter avec eux leur armure et leur bouclier dès lors qu'ils avaient perdu monture et bête de somme. La broigne n'est pas parfaite en termes de protection, on a vu qu'un coup de lance bien assené pouvait la percer, mais elle assurait vraisemblablement une protection appréciable contre les flèches des petits arcs turcs, ainsi que contre les coups d'épées. Le visage, les mains et les jambes ne sont toutefois que peu, ou pas, protégés. Le cheval, il n'est peut-être pas inutile de le signaler, ne dispose d'aucune protection, surtout si le climat est particulièrement chaud. C'est en ce sens que l'image du croisé cuirassé se révèle un mythe: une volée de flèches pouvait très bien arrêter une charge de cavalerie, même si les cavaliers eux-mêmes étaient convenablement protégés.

\section{- Casques}

Autant la question des armures est difficile, autant celle des casque est facile à régler car des exemplaires ont été retrouvés. Il s'agit de casques coniques d'une seule pièce avec nasal rajouté. Un cercle métallique faisait le tour du casque pour en assurer la rigidité et renforcer la protection du combattant. C'était en effet ce cercle qui avait le plus de chance d'être touché par un coup d'épée assené horizontalement. Comme pour l'armure, la protection apportée n'est que relative, un coup d'épée vigoureusement assené pouvant fendre le casque.

\section{- Boucliers}

Les boucliers longs et plats généralisés à la fin du $\mathrm{XI}^{\mathrm{e}}$ siècle étaient peut-être une innovation des Normands. En effet, la première apparition datée de ce type de bouclier se trouve sur la tapisserie de Bayeux, où il équipe les troupes de Guillaume le Conquérant, alors que les Saxons sont protégés par les boucliers ronds et creux

\footnotetext{
${ }^{52}$ Guillaume de Poitiers, Histoire de Guillaume le Conquérant, éd. R. Foreville, Paris, 1952, p. 169 .
} 
qui étaient de rigueur aux siècles précédents. Ces boucliers ronds, mais cette fois plats, selon toute apparence, se rencontrent également figurés dans la Bible de Rho$\mathrm{da}$, à côté de boucliers longs ${ }^{53}$. L'ensemble de l'équipement des piétons et cavaliers de la fin du XI ${ }^{e}$ siècle se retrouve dans une enluminure de la Guerre de Juifs de Flavius Josèphe, provenant de l'abbaye de Saint-Gall (fig. 11). On a vu à propos de l'usage de la lance que le bouclier pouvait être percé. En revanche, il off́re une bonne protection contre les flèches, et sa forme oblongue met la cuisse à l'abri des coups pas trop violents ni trop précis.

\section{Machines de guerre}

\section{- Tours de siège}

Trois tours mobiles furent construites au cours de la première croisade, une à Maara et deux à Jérusalem. Ces engins furent appelés châteaux, et il y a tout lieu de croire qu'ils furent effectivement réalisés sur le modèle des donjons de bois qui étaient alors édifiés en Europe. Munis de quatre roues, ils comprenaient un seul étage, placé à une hauteur telle qu'il puisse surplomber les murs de la ville assiégée. Placés dans cette tour, des chevaliers bombardaient les défenseurs avec de grosses pierres, d'autres les attaquant à la lance. Il ne semble pas que ces tours aient été munies des ponts mobiles que l'iconographie à la manière de Viollet-le-duc se plaît à nous montrer. Il est très net, dans le cas des chevaliers montés dans la tour à Maara, qu'ils ne servirent qu'à nettoyer les remparts de leurs défenseurs, à coups de lances, pendant que d'autres croisés cherchaient à escalader les remparts au moyen d'échelles. Ce n'est d'ailleurs qu'en sapant les murs que les croisés réussirent cette fois-ci à décourager les défenseurs et à les faire abandonner la muraille, avant de pouvoir entrer dans la ville et d'en massacrer quasi totalement la population. Lors du siège de Jérusalem, deux tours furent construites, une au nord, sur ordre de Godefroy de Bouillon, l'autre au sud, sous les ordres de Raymond de Saint-Gilles. La tour sud, que les Provençaux avaient péniblement réussi à mener aux pieds des remparts après avoir comblé le fossé au terme de trois jours de travail, fut incendiée avant qu'elle ait pu servir à quelque chose, et les troupes de Godefroy ayant alors déjà commencé de pénétrer dans la ville, Raymond de Saint-Gilles en fut réduit à négocier son entrée dans Jérusalem avec l'émir Ifthikar-Edaulé, réfugié dans la tour de David, contre sa promesse de lui garantir la vie sauve. Du côté de la tour nord, il est aussi mentionné, quoique peu clairement, l'action parallèle de ceux qui combattent postés sur le château de bois et de ceux qui escaladent les murs de la ville. Parmi eux étaient Liétaud et Engilbert, deux chevaliers originaires de Tournai, qui furent les premiers à pénétrer dans Jérusalem.

\section{- Autres machines}

Les croisés n'utilisèrent pas, semble-t-il, de machine durant leur progression vers Jérusalem. Il y avait à cela une raison bien simple : les machines de jet n'étaient plus connues en Occident, et elles ne sont d'ailleurs pas attestées dans l'iconogra-

\footnotetext{
${ }^{53}$ Paris, Bibliothèque Nationale, ms. lat. 6, t. III, fol. 144.
} 
phie, à l'exception de frondes à manche figurant dans la Bible de Rhoda (fig. 12). Toutefois une machine de jet est décrite par l'Histoire anonyme au siège de Maara, mais dans le camp musulman. D'autres machines, indéterminées, sont mentionnées du côté des croisés au siège de Jérusalem. On ne peut savoir s'il s'agissait de copies de la machine de Maara ou de matériel fournit par les Byzantins. On sait également que les musulmans utilisèrent le feu contre les tours mobiles, à Maara et à Jérusalem, où il réussirent d'ailleurs à incendier celle que Raymond de Saint-Gilles avait fait réaliser au sud de la ville. Mais il est peu probable qu'il s'agisse du véritable feu grégeois, Ousâma précisant qu'on bombardait l'adversaire avec des cruches remplies de naphte. Dans les autres cas, les croisés utilisèrent des échelles ou tentèrent, lorsque le terrain s'y prêtait, de saper les murs. Toutefois, faute de machines de siège dignes de ce nom, le nombre des villes véritablement prises d'assaut fut faible, la plupart étant prise par trahison, par composition, ou à la suite de leur abandon par la population. Ce n'est qu'à partir du XIII siècle que les occidentaux réutilisèrent couramment des machines de siège.

\section{Les points de ralliements}

\section{Les étendards}

La bataille d'Ascalon fut, peut-être davantage que la prise de Jérusalem, le couronnement de la première croisade. A Jérusalem, les croisés avaient espéré que les murs tomberaient d'eux-mêmes dès qu'ils en auraient fait le tour, comme les murs de Jéricho étaient tombés devant l'armée de Josué. Mais le miracle n'avait pas eu lieu, à la grande consternation des croisés. A la bataille d'Ascalon, toutefois, l'intervention de trois cavaliers blancs de grande taille fut bel et bien considérée comme un miracle, une aide apportée par les saints combattants eux-mêmes. L'enjeu était d'ailleurs considérable, et il était à craindre que l'armée égyptienne ne bloquât les croisés dans Jérusalem comme celle de Kerboga les avait assiégés dans Antioche. La victoire complète remportée sur les troupes du vizir Al-Afdal était donc aussi un grand soulagement pour ceux qui avaient connu les affres d'Antioche. Enfin, Ascalon est la dernière bataille où figurent encore tous les grands chefs de l'expédition : peu après la bataille, estimant que leur pélerinage était accompli, Robert Courte-Heuse et Robert de Flandres rentrèrent chez eux avec leurs troupes.

Si Ascalon fut le point d'orgue de la croisade, la prise de l'étendard du vizir par Robert Courte-Heuse fut celui de la bataille. Placé sur une hauteur, entouré de troupes, resplendissant d'or et d'argent, il attirait l'attention de tous, et sa description se retrouve dans tous les récits du combat. Et d'abord dans l'Histoire anonyme de la première croisade (chapitre 39) :

Comes autem de Nortmannia cernens admirabilii standarum habentem quoddam pomum aureum in summitate haste... nostri autem acceperunt ejus stantarum quod comparavit comes de Nortmannia XX marchas argenti et dedit patriarche in Dei honorem Sanctique Sepulchri ${ }^{54}$...

\footnotetext{
${ }^{54}$ Histoire anonyme dé la première croisade, éd. Bréhier, pp. 215-216: Mais le comte de
} Normandie, voyant l'étendard de l'émir qui portait un pommeau en or au sommet de sa 
On trouve une description complémentaire dans le Chronicon Hierosolymitanum d'Albert d'Aix :

Longissima hasta argento coperta per totum, quod vocant standart, et quae regis Babyloniae exercitui signum praeferebatur, et circa quam praecipua virtus densabatur ${ }^{55}$.

L'étendard du vizir fut identifié comme un emblème de commandement important parce qu'il correspondait à des enseignes existantes en Occident: le roi Robert le Pieux (996-1031) avait également comme enseigne une lance couverte d'argent: Invenit ibi suam lanceam a conjuge gloriosa bene argento paratam ${ }^{56}$. Cette enseigne royale figurant parmi les musulmans fut probablement identifiée comme étant un étendard par référence à la Chanson de Roland, du moins dans la version du manuscrit d'Oxford (c. 1070) :

3267 : Et l'estendard Tervagant et Mahum.

3550 et sq. : Et le dragon et l'enseigne le roi

Baligant veit sun gunfanun cadeir

Et l'estandart Mahumet remaneir.

En fait il ne s'agit pas d'un mot d'origine arabe, l'étendard étant cité pour la première fois comme une enseigne militaire dans un texte anglais de Mathieu de Westminster (c. 1016) : Relicto loco qui ex more erat inter draconem et standardum, cuccurit in aciem prima ${ }^{57}$. Auparavant, le mot étendard désignait un candélabre, et c'est sans doute par analogie de forme entre ces deux objets, tous deux composés d'une hampe métallique surmontée d'un pommeau rond, que le premier vint à prendre le nom du second:

Resplendet candelabra, nitet pumorum rotunditas, fulgit recentari colique varietas nec desunt patenae sacris propositionis panibus praeparatae, adsunt et stantarii magnis cereorum corporibus abtati ${ }^{\text {s8 }}$.

hampe... les nôtres reçurent son étendard que le comte de Normandie estima à 20 marcs d'argent, et il le donna au patriarche en l'honneur de Dieu et du Saint-Sépulchre...

${ }_{55}$ Albert d'Aix, Chronicon Hierosolymitanum, éd. Migne, op. cit, t. 166, col 503 : Une longue hampe entièrement couverte d'argent, qu'on appelle étendart, enseigne qui était portée en avant des troupes du roi de Babylone, et autour de laquelle sa force principale était rassemblée.

${ }^{56}$ Helgaud de Fleury, Epitome vite regis Roberti Pii, éd. Migne, op. cit., t. 141, col. 903 (c. 1033) : Il trouva là sa lance bien parée d'argent par sa glorieuse épouse.

${ }^{57}$ Cf. Dictionnaire Du Cange, t. 6 : «laissant le lieu qui, suivant la coutume, se trouvait entre le dragon et l'étendard, il court en première ligne"

${ }^{58}$ Vita s. Desideri Cadurcae urbis episcopi, M. G. H., Scriptorum rerum Merovingicorum, p. 576 (c. 800) : Les candélabres resplendissent, la rotondité des pommeaux brille, scintille la variété de vase et de filtre, les patènes pleines d'offrandes consacrées ne font pas défaut, les étendards de cire préparés en grandes masses sont présents. 
On saisit mieux pourquoi c'est Robert Courte-Heuse, fils de Guillaume le Conquérant, qui est probablement le premier à avoir décidé que l'emblème du vizir était un étendard, compte tenu de ce qu'il savait des enseignes anglo-normandes, qui avait été le mieux à même, parmi les croisés, de comprendre l'importance tactique qu'il pouvait y avoir à s'en emparer, répétant l'exploit d'Ogier de Danemark, pour autant, bien sûr, qu'il ait eu connaissance de la Chanson de Roland.

\section{Les tentes}

Du côté des croisés il n'y avait pas d'emblème de ce niveau, car l'empereur Henri IV, les rois d'Angleterre et de France ne participaient pas à la croisade, étant alors excommuniés. Les ducs et comtes ne portaient que des vexilla sans marque particulière, les armoiries n'existant pas encore. Pour marquer un point de ralliement dans la bataille, on voit les croisés dresser les tentes, dès le combat de Dorylée. Ce fait n'était peut-être pas propre aux seuls croisés, mais était vraisemblablement répandu dans tout l'Occident, du moins lorsqu'il n'y avait pas à proximité un point fortifié ou une enseigne jouant ce rôle. Les Allemands se rassemblaient autour de l'Aigle impériale et du gundfano de l'empereur, les Anglais, selon Mathieu de Westminster, entre l'étendard et le Dragon, qu'adoptèrent par la suite les AngloNormands. Les Français, après s'être rassemblés autour d'une lance argentée, se regroupèrent à partir de 1124 autour de l'Oriflamme. Les croisés auraient pu, après la prise de Jérusalem, utiliser à cet usage la sainte Croix, mais cette relique ne joua pas à proprement parler de rôle militaire. Ainsi lors de la fameuse bataille de Hattin (1187), Saladin ne tint sa victoire pour assurée que lorsqu'il vit s'effondrer la tente du roi de Jérusalem, Guy de Lusignan. La prise de la sainte Croix, portée au milieu de cette même bataille, n'avait pas eu la même importance ${ }^{59}$.

\section{Conclusion}

Il ne semble pas possible de dire que l'armement des croisés fut supérieur dans l'ensemble à celui de leurs adversaires, et même si une étude de l'équipement musulman est encore nécessaire pour justifier cette idée, on peut abandonner l'idée généralement répandue du croisé blindé face au musulman en tunique légère. Les cuirasses existaient de part et d'autre, mais l'équipement était de qualité variable selon les fortunes de chacun. L'armement n'était pas non plus uniforme dans le temps, la perte d'un animal de bât pouvant entraîner pour un chevalier la nécessité de jeter cotte de mailles et bouclier.

Jusqu'à Antioche, le nombre des croisés leur donna la puissance d'un rouleau compresseur, après, il est évident que leurs qualités militaires furent davantage mises à contribution, même si la terreur qu'ils inspirèrent et la division du camp musulman ne les mirent jamais dans le cas d'affronter des ennemis nettement supérieurs numériquement. Il ne faut d'ailleurs pas négliger les effets d'un probable aguerrissement des croisés au cours de leur progression, qui pourrait se marquer également

${ }^{59}$ J. Richard, «La bataille de Hattin», Les Croisades, Paris, 1988, p. 193. 
dans l'emploi progressif de machines de guerre, plus nombreuses et plus variées après Maara. Il est incontestable toutefois que les croisés eux-même eurent l'impression d'une véritable supériorité militaire, ce qui les amena parfois jusqu'à l'outrecuidance et leur valut des revers extrêmement sévères. La réussite des premiers croisés est pourtant finalement due à la désorganisation des musulmans et à leur propre cohésion. Lorsqu'un siècle plus tard, les Etats latins furent à leur tout divisés par des querelles de chefs face à un Moyen-Orient unifié - relativement - par Saladin, ce fut la déroute. En cette affaire, la qualité de l'armement ne joua certainement qu'un rôle relatif, d'autant plus que les prises de guerre, les cadeaux et le commerce introduisirent dans chaque camp les armes de l'adversaire. La victoire des croisés n'est pas celle des cavaliers bardés de fer de Charlemagne sur les Lombards du roi Didier; elle est celle de la cohésion des chevaliers francs sur un Orient incapable de s'unifier. C'est finalement l'unification des adversaires des croisés, patiemment et difficilement réalisée, qui aura raison des Etats latins d'Orient.

Olivier Bouzy 


\section{Illustrations}

fig. 1 : Saint Michel, chapiteau du portail ouest, piédroit de droite, église de Perrecylès-Forges (Saône-et-Loire), cl. C.E.S.C.M. Poitiers.

fig. 2 : Mort d'Absalom, chapiteau du nord de la nef, église de la Madeleine, fin $\mathrm{XI}^{\mathrm{e}}$ début XII siècle, Vézelay (Yonne), cl. C.E.S.C.M. Poitiers.

fig. 3 : Meurtre de l'Amalécite $\left(2^{\text {nd }}\right.$ livre de Samuel, 1,15$)$, chapiteau du nord de la nef, église de la Madeleine, fin $\mathrm{XI}^{\mathrm{e}}$-début $\mathrm{XII}^{\mathrm{e}}$ siècle, Vézelay (Yonne), cl. C.E.S.C.M. Poitiers.

fig. 4 : Martyre de saint Blaise, abside du bas-côté nord, château des moines (ancien prieuré), XI ${ }^{e}$ siècle, Berzé-la-Ville (Saône-et-Loire), cl. C.E.S.C.M. Poitiers.

fig. 5 : Deux chevaliers combattant, frise de la façade ouest de l'ancienne collégiale, $\mathrm{XI}^{e}$ siècle, Andlau (Bas-Rhin), cl. C.E.S.C.M. Poitiers.

fig. 6 : Mattathias tue les Juifs sacrifiant aux autels païens, croquis d'après ms. du Livre des Machabées, II, 24, 5. Scriptorium de Saint-Gall, première moitié du IX siècle, Leyde, Bibliotheek der Rijksuniversiteit, codex Peruzoni 17, fol. 9. cf. J. Hubert, J. Porcher, W.F. Volbach, L'Empire carolingien, Paris, 1968, fig. 163, p. 177.

fig. 7 : Pommeau d'une épée trouvée dans la Vilaine à Rennes, Musée du Mans, $\mathrm{n}^{\circ}$ inv. 455 , cl. Klinka.

fig. 8 : La sainte famille partant en Egypte, Chapiteau déposé provenant de la salle capitulaire de la cathédrale Saint-Lazarre, début XII ${ }^{\mathrm{e}}$ siècle, Autun (Saône-et-Loire), cl. C.E.S.C.M. Poitiers.

fig. 9 : Epieu, longueur $60 \mathrm{~cm}$, largeur lame $5,5 \mathrm{~cm}$, trouvé dans la Saône à Port-deBoz (Ain), Chalon-sur-Saône, Musée Denon, inv. 83.21.2, cf. catalogue exposition Du silex à la poudre, quatre milles ans d'armement en val de Saône, Montagnac, $1991, n^{\circ} 164$, fig. 115 , p. 134.

fig. 10 : Epieu, longueur $79,2 \mathrm{~cm}$, largeur lame $14,5 \mathrm{~cm}$, trouvé dans le Doubs à Sanière (Saône-et-Loire), Chalon-sur-Saône, Musée Denon, inv. 82.10.16, $c f$. catalogue exposition $\mathrm{Du}$ silex à la poudre, quatre milles ans d'armement en val de Saône, Montagnac, 1991, n²22, fig. 147, p. 147.

fig. 11 : guerriers, croquis d'après ms. de Flavius Josèphe, Guerre des Juifs, Scriptorium de Saint-Gall, $\mathrm{XI}^{\mathrm{e}}$ siècle, B.A. ms. 843, fol. 77.

fig. 12 : Bataille d'Eleasa, mort de Judas Machabée, croquis d'après ms. du Livre des Machabées, IX, 17, 19, Bible de Saint-Pierre-de-Rhoda, $\mathrm{XI}^{e}$ siècle, Paris, Bibliothèque Nationale, ms. lat. $6, \mathrm{t}$. III, fol. $144 \mathrm{v}^{\circ}$, cl. B.N. 

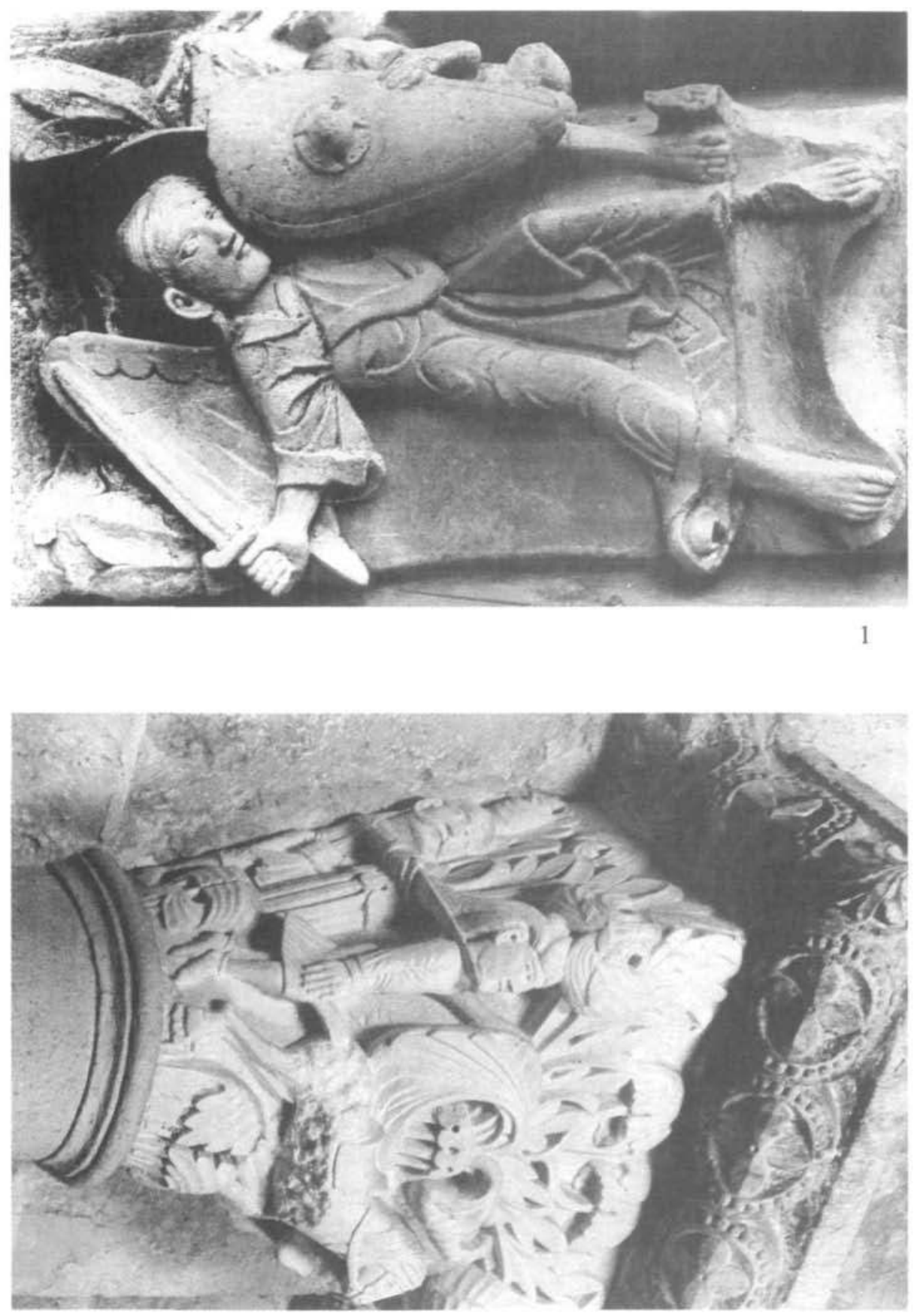

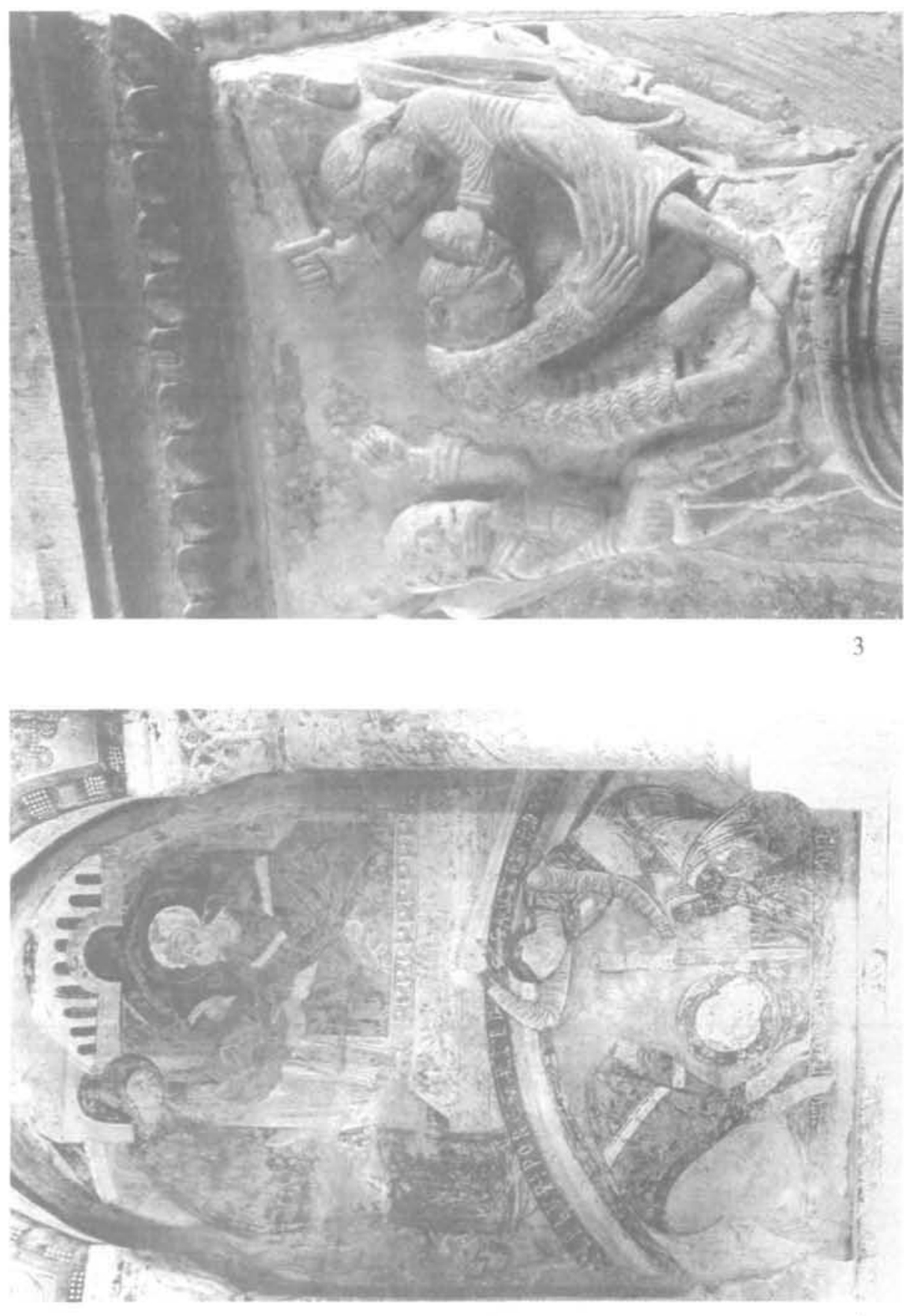

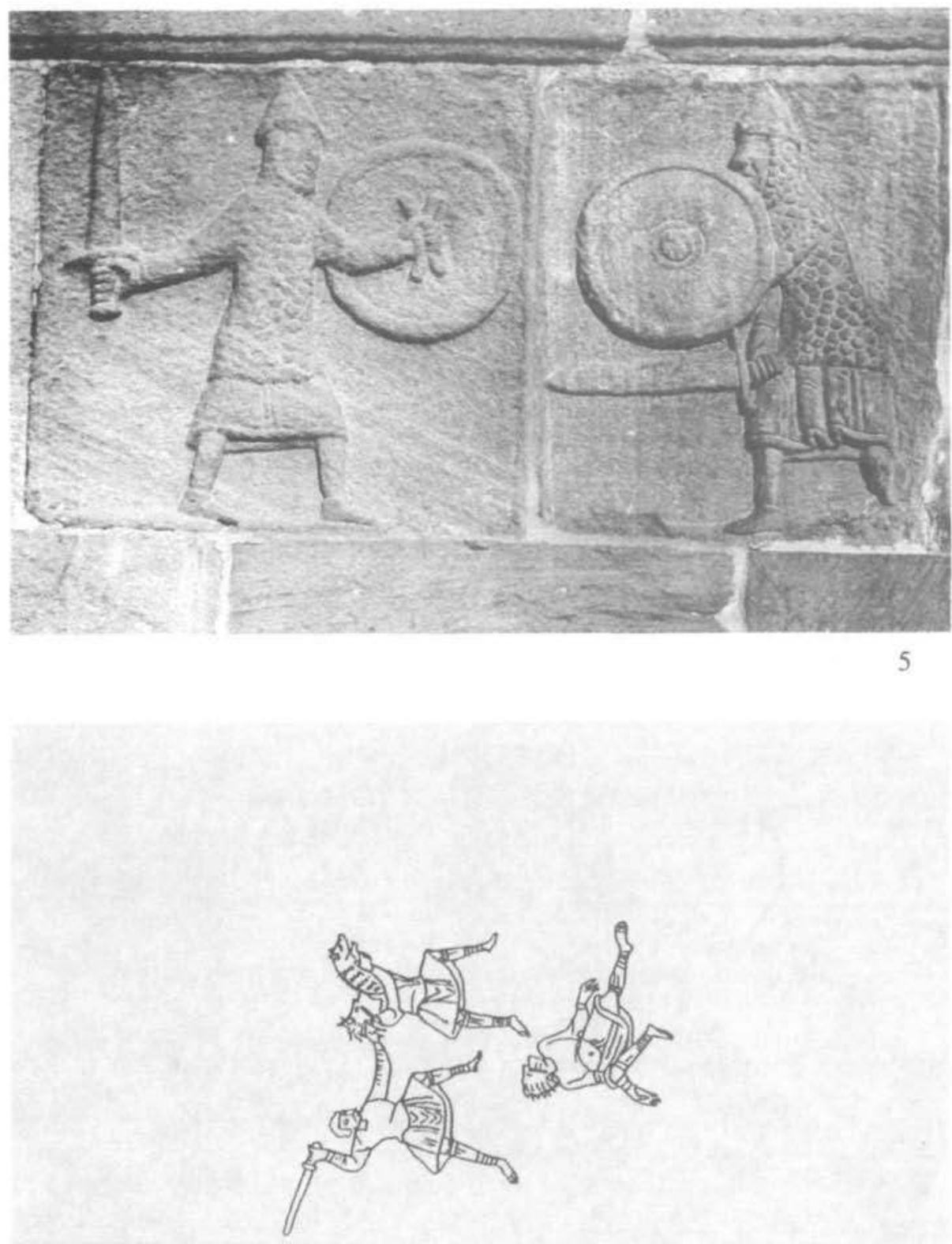

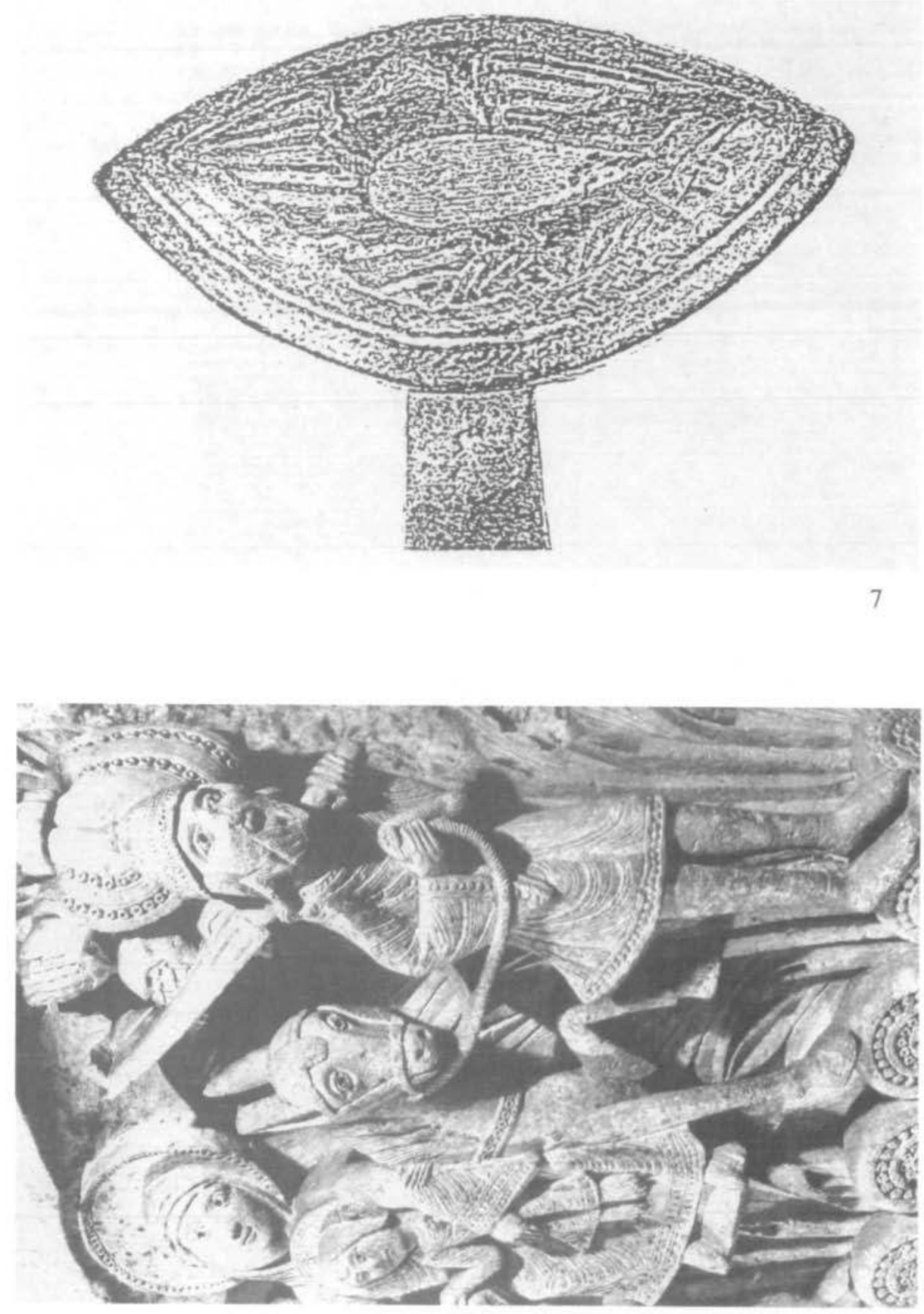

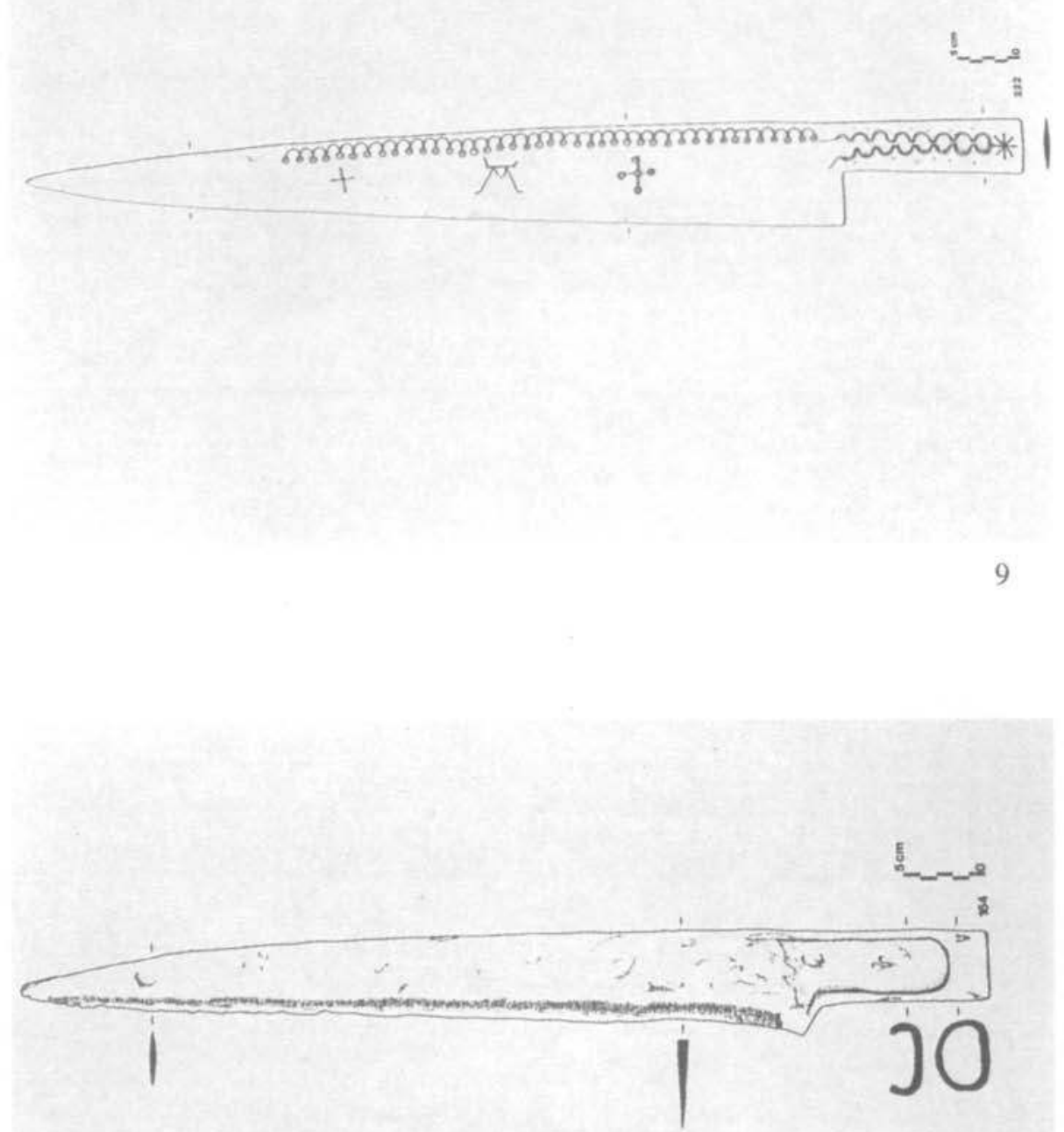

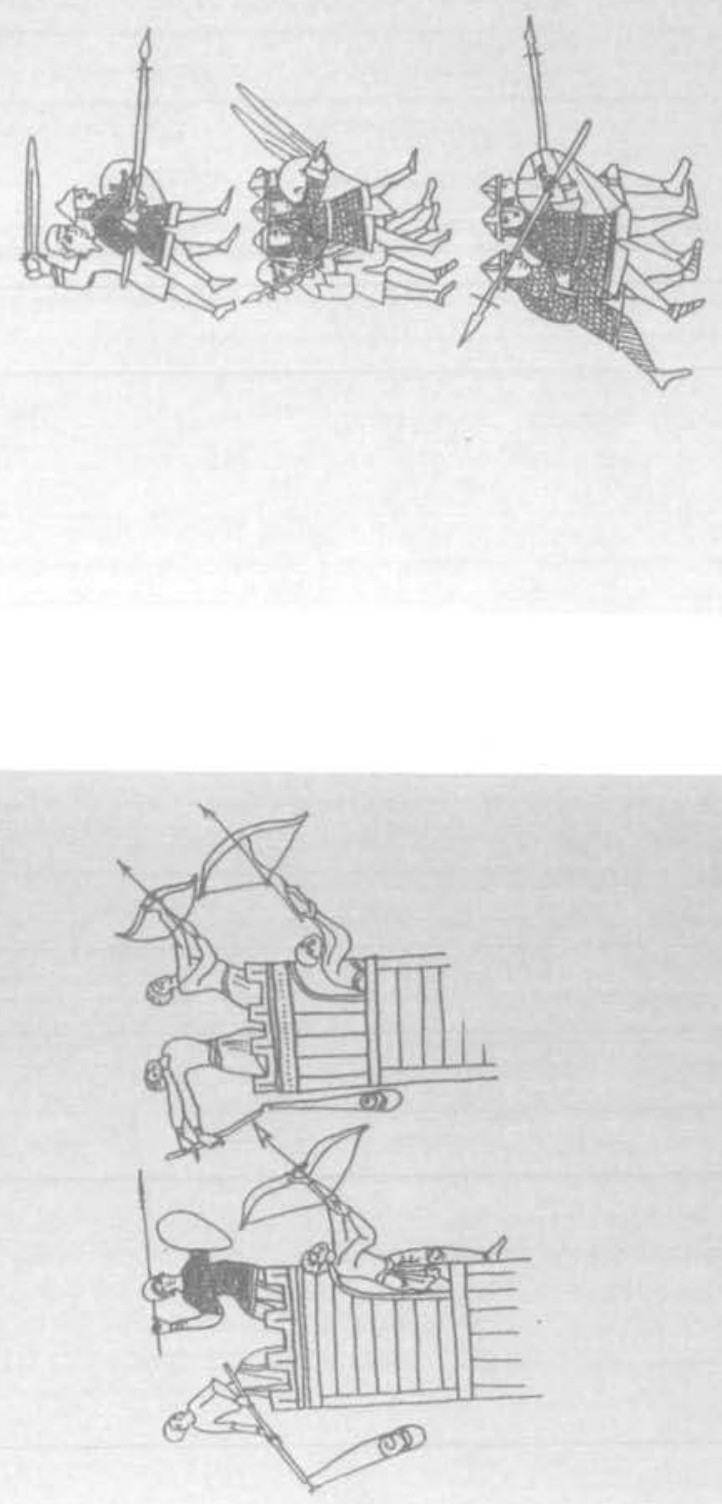